Article

\title{
Impact of Mixed Infections of Gut Parasites Lotmaria passim and Nosema ceranae on the Lifespan and Immune-related Biomarkers in Apis mellifera
}

\author{
Nolberto Arismendi ${ }^{1,2, *}$, Solange Caro ${ }^{2}$, María Paz Castro ${ }^{2}$, Marisol Vargas ${ }^{2}$ (D), \\ Gustavo Riveros ${ }^{2}$ and Tomas Venegas ${ }^{2}$ \\ 1 Austral Biotech Research Center, Faculty of Science, Universidad Santo Tomás, \\ Av. Ramón Picarte, Valdivia 1130, Chile \\ 2 Laboratories of Virology and Bee Pathology, Facultad de Agronomía, Universidad de Concepción, \\ Av. Vicente Méndez, Chillán 595, Chile; solangecaro@udec.cl (S.C.); mcastrot@udec.cl (M.P.C.); \\ marisolvargas@udec.cl (M.V.); gustavoriveros@udec.cl (G.R.); rovenegas@udec.cl (T.V.) \\ * Correspondence: narismendi@santotomas.cl
}

Received: 12 June 2020; Accepted: 3 July 2020; Published: 8 July 2020

check for updates

\begin{abstract}
Lotmaria passim currently appears to be the predominant trypanosome in honey bees worldwide. Although, the specific effects of L. passim by single or mixed with other gut parasites such as Nosema ceranae on honey bees' health is still unclear. We consequently measured bees' survival, parasite loads, the expression of antimicrobial peptides (AMPs) and vitellogenin gene. Thus, (1) bees naturally infected with L. passim, (2) healthy bees inoculated with Nosema ceranae, (3) bees naturally infected with L. passim and inoculated with $N$. ceranae and (4) healthy bees (control) were maintained under controlled conditions. Honey bees infected with $N$. ceranae or with mixed infections of L. passim and N. ceranae had significantly lower survival rates than the control group at 20 days post-inoculation (dpi). A competitive suppression was also detected, provided that the L. passim load was significantly affected by the presence of $N$. ceranae at 15 dpi. Expressions of the AMPs defensin and hymenoptaecin rapidly (two hours post-inoculation) increased in bees infected with $N$. ceranae and mixed infections. However, this effect was not continuous. In fact, expressions of abaecin, defensin, hymenoptaecin and vitellogenin decreased drastically at $15 \mathrm{dpi}$ in bees with both single and mixed infections. The decrease in the expression of AMPs and vitellogenin throughout this period was consistent with the reduced survivals observed in this study, indicating that mixed infections of L. passim and N. ceranae, and even into a scenario of competition between them, may have a synergic effect on the survival and immune-related gene expressions (biomarkers) of worker bees.
\end{abstract}

Keywords: AMPs; vitellogenin; lifespan; trypanosome; microsporidium; honey bee

\section{Introduction}

There are currently two species of trypanosomes that invade the gut of Apis mellifera (L.); Crithidia mellificae Langridge and McGhee and Lotmaria passim Evans and Schwarz. Lotmaria passim was described only a few years ago [1] and is now considered the most predominant trypanosome of honey bees in Asia, Europe and South and North Americas, wherein C. mellificae is considered infrequent or absent [1-4]. Trypanosomes in bee species have become more relevant in recent years, since they have proven to have negative effects on behavior, physiology, the immune system and the lifespan of the hosts [5-8]. However, the specific effects of L. passim on honey bee survival, immunity and host physiology is still unclear. On the other hand, the gut parasite Nosema ceranae Fries (Microsporidia) has been more thoroughly considered as a factor contributing to bee losses, either alone or in combination with other parasites [9-12]. Nosema ceranae was first reported in European honey bees around 10 years 
ago in Europe and Asia $[13,14]$. Since these first reports, N. ceranae has now become one of the most prevalent honey bee microsporidia worldwide [15]. This parasite has been implicated in honey bee immunosuppression, the reduction of the host lifespan and physiological and behavioral changes, as well as negatively affecting the productivity of honey bee colonies [10,16-18]. Nosema ceranae has also been implicated in the global phenomenon of colony loss, at least in Spain [9].

It is not unusual that multiple parasite genotypes, either distinct strains of the same species or different species, coinfect a single host [19], especially in social insects, where the transmission of beneficial or pathogen agents may be facilitated by social interactions [20]. Pathogen mixed infections may interact in different ways within the host; in some cases, pathogens can act independently of one another; positive interactions are also possible, when one proliferates due to the presence of the other, and finally, negative interactions can also occur when pathogens suppress one another [21]. Experimental evidence has shown a synergistic effect when mixed infections of $N$. ceranae and other honey bee pathogens have been observed [12]. These mixed infections may alter local and systemic immune gene transcriptions, the survival and, in some cases, the learning and memory capabilities of honey bees $[7,11,22]$. However, there is no experimental evidence related to how a mixed infection of N. ceranae and L. passim could affect honey bee colonies. Some current reports have shown that mixed infections of these pathogens are not common (no more than $2 \%$ ), in spite of the fact that they have been detected in high prevalence in single infections in honey bee hives [3,23], suggesting a possible competition between these two parasites. Furthermore, such mixed-species infections may influence the host immune responses, including genes that elicit antimicrobial peptides (AMPs) $[7,16]$. AMPs are considered as a key component in insect innate immunity, and four families of AMPs (abaecin, apidaecin, defensin and hymenoptaecin) have been described in honey bees [24,25]. There is evidence that honey bee immune responses (AMPs) are dynamic in transcriptional intensity and diversity over short or long periods of time-for instance, after N. ceranae infections [7,16]. Furthermore, single and mixed infections of parasites may also affect other relevant components associated with immune senescence, such as the phospholipoglycoprotein vitellogenin [26]. In worker honey bees, vitellogenin play multiple roles; it is important in bee behaviors associated with nursing and foraging activities but is also essential for bees' immune responses, oxidative stress resilience (antioxidant properties) and longevity [27-29]. Therefore, AMPs and vitellogenin genes could be used as biomarkers for honey bee health and physiology, since their expression can be affected by biological stressors such as varroa mites, microsporidia, trypanosomes and viruses [16,30-32]. New research is thus required in order to determine the effects of L. passim alone or in combination with $N$. ceranae on honey bees' health and, also, to determine whether the interaction between these two gut pathogens has a synergistic effect, a competitive effect or no effect at all. To address the possible impacts of single and mixed infections of these two gut parasites on honey bees' lifespans and their expression of AMPs and the vitellogenin gene $(\mathrm{Vg})$, we monitored the signaling of AMPs and oxidative stress related-gene $(\mathrm{Vg})$ expressions over a 20-day period post-inoculation. We also quantified the parasite loads and bee survival rates of honey bees with a basal level of L. passim infection, as well as bees (both healthy and L. passim-infected) that were inoculated with $N$. ceranae spores after emergence.

\section{Materials and Methods}

\subsection{Biological Assays in Honey Bees}

Provided that we have detected L. passim in honey bee workers of different ages and developmental stages [33] and, also, the difficulty to obtain pure cultures of L. passim cells, we decided to evaluate the possible effects of $N$. ceranae in bees from colonies with a basal level $\left(1.0 \pm 0.6 \times 10^{3}\right.$ cell per bee) of L. passim in addition to uninfected honey bees. Furthermore, this experiment gave the opportunity to evaluate the effects of the occasional infection of $N$. ceranae in bees that were previously infected with L. passim. Additionally, it is necessary to mention that, at the beginning of the season (spring), all the queens in the colonies were replaced by new queens (A. mellifera carnica) that were bought at 
the same honey bee queen supplier company (https://www.jphmielapicultura.cl/) in order to ensure similar queen genetics in the colonies and, thus, to avoid a genetic effects of the queen on worker bees used in the experiment.

The health status of colonies $(n=40)$ was previously determined by molecular techniques according to Vargas et al. [3] (Table S1, Supplementary Materials). Then, brood combs with capped worker bees were removed from uninfected ( $\mathrm{n}=1$ per colony, 4 colonies) and L. passim-infected colonies ( $\mathrm{n}=1$ per colony, 4 colonies) and maintained under controlled conditions in a rearing room $\left(30{ }^{\circ} \mathrm{C} \pm 1.0\right.$ and $60 \% \pm 3.0 \%$ relative humidity $(\mathrm{RH})$ ). Afterwards, newly emerged worker bees were carefully collected from the brood comb and randomly confined in plastic cages (base $=8 \mathrm{~cm}$ diameter, mouth $=10 \mathrm{~cm}$ diameter and height $=15 \mathrm{~cm}$ ) to reduce the effects of the colony genetics on the experiments. The bees were supplied with sucrose syrup $60 \%(\mathrm{w} / \mathrm{v})$ for $24 \mathrm{~h}$. After this time, the live bees were used for assays. Therefore, to test the effects of L. passim alone or its synergistic effects due to a mixed infection with $N$. ceranae, the treatments were as follows: (1) bees naturally infected with L. passim (Lp), (2) healthy bees inoculated with $N$. ceranae $(\mathrm{Nc})$, (3) bees naturally infected with L. passim and inoculated with $N$. ceranae (mixed-species infection) and (4) uninfected honey bees (control treatment). In the bees that had to be inoculated with $N$. ceranae, the spores were purified from N. ceranae-infected colonies according to the methodology described by Fries et al. [34]. Thus, the bees were starved for $3 \mathrm{~h}$ and were then individually infected with $N$. ceranae spores $\left(1.0 \pm 0.3 \times 10^{5}\right.$ spores per bee), which were mixed in $60 \%$ sucrose syrup ( $5 \mu \mathrm{L}$ per bee) according to Porrini et al. [35]. Those bees that did not consume the total amount of $N$. ceranae spore suspensions were discarded from the assay. Then, 75 worker bees ( 2 days old) were maintained in plastic cages $(4$ replicates per treatment) containing $3 \mathrm{~g}$ of pollen substitute (soybean meal $(18 \%)$, curbiculated pollen $(10 \%)$, corn flour $(6 \%)$, wheat flour $(6 \%)$, potato starch $(2 \%)$, canola oil $(0.2 \%)$ and sucrose $(57.8 \%)$ mixed in distilled water to make a patty) and supplied with $60 \%$ sucrose syrup ad libitum [36]. Dead bees were counted daily and removed from cages until the end of the experiment (20 days total) for a survival analysis. Furthermore, five bees were removed from each cage after $2 h$, as well as 5, 10 and 15 days post-inoculation (dpi) in all treatments for a molecular analysis.

\subsection{Nucleic Acid Extraction, cDNA Synthesis and Real-Time PCR}

Honey bee abdomen samples ( 5 abdomens per replicate) were homogenized with $5 \mathrm{~mL}$ of phosphate-buffered saline (PBS 1X) with a mortar and pestle in cold conditions. Then, $200 \mu \mathrm{L}$ of the abdomen extract was used for RNA extraction according to the instructions provided by the E.Z.N.A. Total RNA Kit I (OMEGA, Bio-Tek Inc., Atlanta, GA, USA). However, the TRK lysis buffer included in the RNA Kit I was replaced by $500 \mu \mathrm{L}$ of Trizol ${ }^{\mathrm{TM}}$ Reagent (Invitrogen, Thermo Fisher Scientific, Waltham, MA, USA) in order to improve the RNA isolation.

For the DNA extraction, another $200 \mu \mathrm{L}$ of the abdomen sample extract was ground in $1.5-\mathrm{mL}$ microcentrifuge tubes using sterile plastic pestles with 350- $\mu \mathrm{L}$ control (CTL) buffer following the E.Z.N.A. Insect DNA Kit's instructions (Omega, Bio-Tek, Norcross, GA, USA). DNA and RNA were then quantified (Infinite 200 PRO NanoQuant, Tecan Group Ltd., Männedorf, Switzerland) and stored at $-80^{\circ} \mathrm{C}$.

The RNA extracted was used for first-strand cDNA synthesis, which was performed using the M-MLV reverse-transcriptase enzyme (Invitrogen, Life Technologies, Carlsbad, CA, USA), according to the manufacturer's instructions. The cDNA samples were maintained at $-20{ }^{\circ} \mathrm{C}$ until the real-time PCR (qPCR) analyses were carried out.

In order to quantify the L. passim and N. ceranae loads in infected samples (with both single and mixed-species infections), a real-time PCR (qPCR) was carried out with specific primers capable of amplifying the ribosomal RNA gene in both gut parasite species (Table 1). Additionally, to detect and quantify the possible changes in the expression of the immune-related genes that encoded AMPs such as abaecin, defensin (defensin-1) and hymenoptaecin, as well as vitellogenin $(\mathrm{Vg})$, we used primers that have been previously published (Table 1). Thus, the PCR reactions were carried out in $15 \mu \mathrm{L}$ 
(4 biological replicates per treatment and two technical replicate per sample), containing $20 \mathrm{ng}$ of cDNA, 1X of KAPA SYBR FAST Universal 2X qPCR Master Mix (Kapa Biosystems, Wilmington, MA, USA), $530 \mathrm{nM}$ of forward primer, $530 \mathrm{nM}$ of reverse primer and enough sterile-filtered molecular grade water to reach the total $15 \mu \mathrm{L}$. The thermal conditions were achieved with one cycle at $95^{\circ} \mathrm{C}$ for $10 \mathrm{~min}$, followed by 40 cycles at $95^{\circ} \mathrm{C}$ for $15 \mathrm{~s}, 60^{\circ} \mathrm{C}$ for $15 \mathrm{~s}$ and $72{ }^{\circ} \mathrm{C}$ for $15 \mathrm{~s}$. A dissociation analysis was conducted after all of the amplifications were completed in order to detect the primer dimmers and the unspecific amplicons. Thus, the data regarding the pathogen loads were reported as cell or spore equivalents per bee, according to Tritschler et al. [23]. Similarly, the data concerning immune-related genes were reported as relative expressions after normalization with an endogen gene ( $\beta$-actin), according to Pfaffl [37].

Table 1. List of gene-specific primers used in the real-time PCR (qPCR) analysis.

\begin{tabular}{|c|c|c|c|c|c|c|}
\hline Primer Name & Sequences $\left(5^{\prime}-3^{\prime}\right)$ & Gene & Size (bp) & Efficiency (\%) & Target & References \\
\hline Lp5F & GGCGTCCGTGATTTTTACTGTGACTA & SSU rRNA & 186 & 103 & Lotmaria passim & This study \\
\hline Lp5R & ACCACAAGAGTACGGAATGCGAAAG & & & & & \\
\hline Nc841F & GAGAGAACGGTTTTTTGTTTGAGA & SSU rRNA & 147 & 102 & Nosema ceranae & [38] \\
\hline Abaecin-F & CAGCATTCGCATACGTACCA & Abaecin & 130 & 100 & AMP abaecin & [24] \\
\hline Abaecin-R & GACCAGGAAACGTTGGAAAC & & & & & \\
\hline Defensin-F & TGTCGGCCTTCTCTTCATGG & Defensin-1 & 201 & 96 & AMP defensin & [39] \\
\hline VgMC-F & AGTTCCGACCGACGACGA & $\mathrm{Vg}$ & 63 & 94 & Vitellogenin precursor & [18] \\
\hline VgMC-R & TTCCСTCCCACGGAGTCC & & & & & \\
\hline B-actin-F & ATGCCAACACTGTCCTTTCTGG & $\beta$-actin & 151 & 96 & $\beta$-actin (reference gene) & [39] \\
\hline B-actin-R & GACCCACCAATCCATACGGA & & & & & \\
\hline
\end{tabular}

${ }^{1}$ New primers used for Lotmaria passim detection and quantification were designed and validated according to the procedure described by Arismendi et al. [2]. AMP: antimicrobial peptides.

\subsection{Data Analysis}

Survival curves of uninfected worker bees, as well as those with single and mixed-species infections were plotted using a Kaplan-Meier estimator considering the live bees at the end of the experiment as censored data. Differences among the survival curves were estimated using a Log-rank test $(p<0.05)$, and $p$-values were corrected $\left(p^{\prime}<0.05\right)$ with the Holm-Bonferroni method [40] for pairwise multiple comparisons with respect to the control group (uninfected bees).

The L. passim and N. ceranae load data $\left(\log _{10}\right)$ in infected honey bees were not normally distributed (Shapiro-Wilk test, $p<0.05)$. Thus, a Mann-Whitney U test $(p<0.05)$ was run to compare the pathogen loads for each time periods ( $2 \mathrm{~h}$ and 5, 10 and $15 \mathrm{~d}$ ) post-inoculation. Control treatments (uninfected bees) were excluded from these analyses because no N. ceranae or L. passim infections were detected in the qPCR.

Statistical differences in the relative expression $\left(\log _{10}\right)$ of AMPs and vitellogenin for each time period post-inoculation for single, mixed-species-infected and uninfected honey bees were estimated by one-way ANOVA. Then, a Dunnett test was run to separate the means in L. passim or N. ceranae-infected and mixed-species-infected bees with respect to the control. All analyses were carried out with STATISTICA 7.0 software (StatSoft, Tulsa, OK, USA).

\section{Results}

Worker bees infected with N. ceranae or bees naturally infected with L. passim and inoculated with $N$. ceranae (bees with mixed-infections) survived for significantly less time than the control group (uninfected bees) (Nc vs. control, Log-rank test value $=2.52, p=0.012\left(p^{\prime}=0.038\right.$ ) and Lp (with Nc) vs. control Log-rank test value $=3.57, p=0.003\left(p^{\prime}=0.010\right)$ ). In fact, bees infected with $N$. ceranae and bees infected with both L. passim and N. ceranae showed survivals circa $40 \%$ and $30 \%$, respectively, whilst the control group showed a survival over 65\% (Figure 1). On the contrary, there was no significant difference (Lp vs. control, Log-rank test value $=0.88, p=0.381$ ) in the survival curve of bees that were naturally infected with L. passim and the control at the end of experiment (20 dpi; Figure 1). 


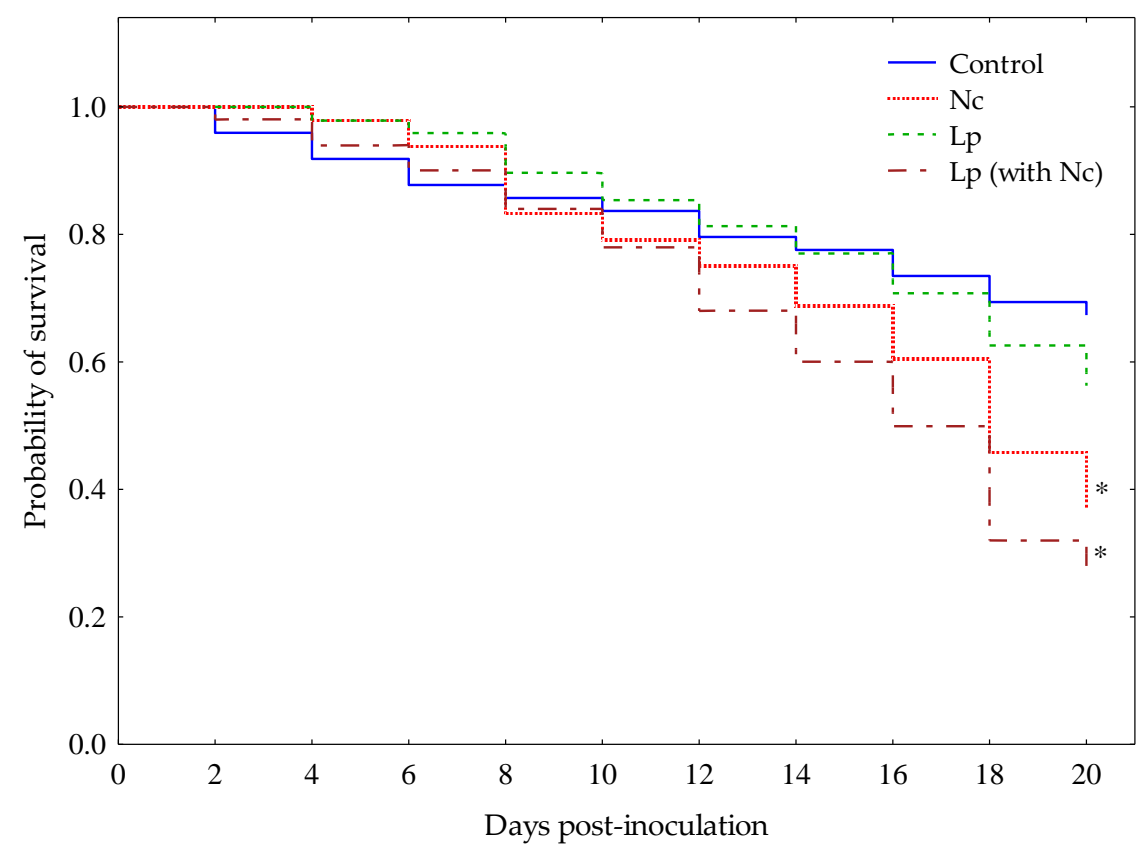

Figure 1. Kaplan-Meier survival curves distribution of worker bees in each experimental group during the 20 days. Each curve represents bees naturally infected with Lotmaria passim (Lp), healthy bees inoculated with N. ceranae $(\mathrm{Nc})$, bees naturally infected with L. passim and inoculated with N. ceranae (Lp (with $\mathrm{Nc}$ ) = mixed-species infection) and uninfected honey bees (control). Asterisks indicate significant differences $(*=p<0.05$ ) of pathogen-infected bees with respect to the control group, according to the Log-rank test with Holm-Bonferroni correction.

The L. passim load in naturally infected honey bees was significantly affected (Mann-Whitney test $\mathrm{U}=0.00, \mathrm{z}=2.31, p=0.021$ ) by the presence of $N$. ceranae after $15 \mathrm{dpi}$; previous to this time, no differences were observed (Figure 2A). On the other hand, the N. ceranae load was not altered by the presence of L. passim in the same host (Figure 2B).

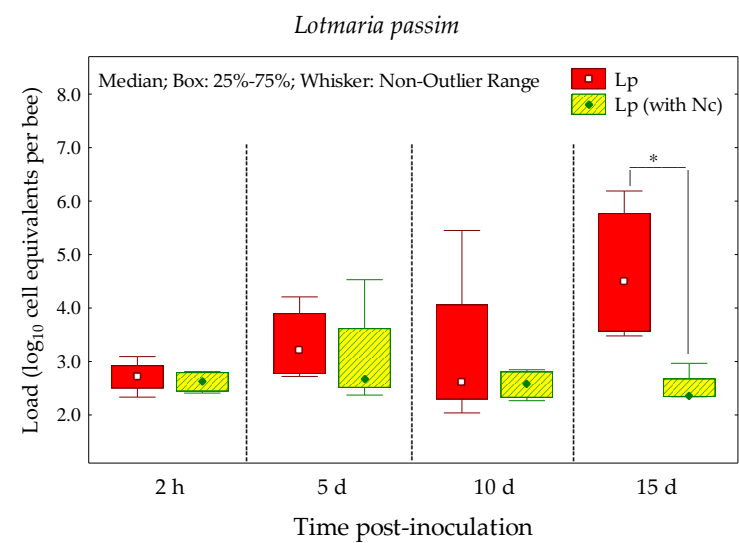

(A)

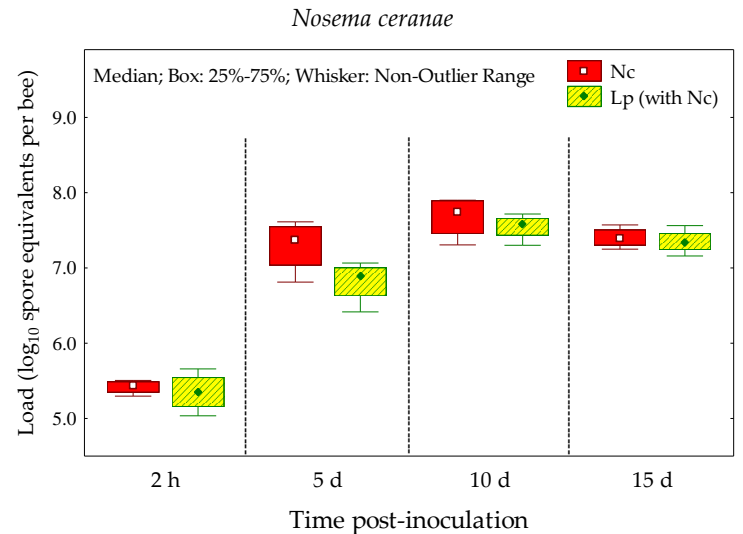

(B)

Figure 2. Lotmaria passim (A) and N. ceranae (B) loads quantified in bees naturally infected with L. passim (Lp) and bees that were L. passim-infected and inoculated with N. ceranae (Lp (with Nc)) at different times post-inoculation. Asterisks in the box plots indicate significant differences $\left({ }^{*}=p<0.05\right)$ of pathogen loads according to the Mann-Whitney U test.

Honey bees infected with N. ceranae, L. passim and those mixed-infected with both pathogens induced significant changes in their relative expressions of genes encoding the abaecin peptide 10 and 
15 days post-inoculation (ANOVA 10 dpi $\mathrm{F}_{(3,12)}=2.34, p=0.047$ and 15 dpi $\mathrm{F}_{(3,12)}=9.77, p=0.002$ ). Thus, bees infected with $N$. ceranae and those with mixed infections of L. passim and N. ceranae had significantly higher abaecin mRNA expressions at 10 dpi compared with the control group. However, the abaecin gene expression was significantly downregulated at $15 \mathrm{dpi}$ in single infections of either L. passim or N. ceranae (Figure 3A). The relative expressions of the genes encoding defensin and hymenoptaecin peptides were also altered significantly in infected bees at $2 \mathrm{~h}$ (ANOVA defensin $\mathrm{F}_{(3,12)}=5.27, p=0.015$ and hymenoptaecin $\left.\mathrm{F}_{(3,12)}=10.09, p=0.001\right)$ and 15 dpi (ANOVA defensin $\mathrm{F}_{(3,12)}=3.53, p=0.049$ and hymenoptaecin $\left.\mathrm{F}_{(3,12)}=4.43, p=0.026\right)$. Defensin and hymenoptaecin mRNA expressions rapidly ( $2 \mathrm{~h}$ post-inoculation (hpi)) increased in bees that were $N$. ceranae-infected and mixed-infected with L. passim and N. ceranae. Following 2 hpi, no changes in the expressions of either of the aforementioned AMPs were observed at 5 and 10 dpi compared with the control group (Figure 3B,C). Nonetheless, a minor yet significant expression of hymenoptaecin was detected at $15 \mathrm{dpi}$ in L. passim-infected and N. ceranae-infected bees (Figure 3C). In the case of Vg gene expression levels, significant differences among the analyzed groups were also found at 5 and 15 dpi (ANOVA 5 dpi $\mathrm{F}_{(3,12)}=4.07, p=0.033$ and $\left.15 \mathrm{dpi} \mathrm{F}_{(3,12)}=14.35, p<0.001\right)$. The $\mathrm{Vg}$ relative expression was significantly lower in bees with mixed infections of L. passim and N. ceranae compared with the control group at $5 \mathrm{dpi}$. This effect was not observed to be significant at $10 \mathrm{dpi}$, but five days later, bees infected with L. passim, N. ceranae and those with both pathogens showed significant reductions in their levels of the $\mathrm{Vg}$ gene expression with respect to uninfected bees (Figure 3D).

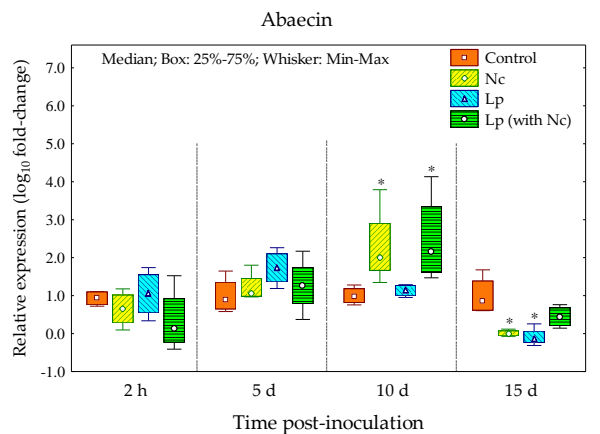

(A)

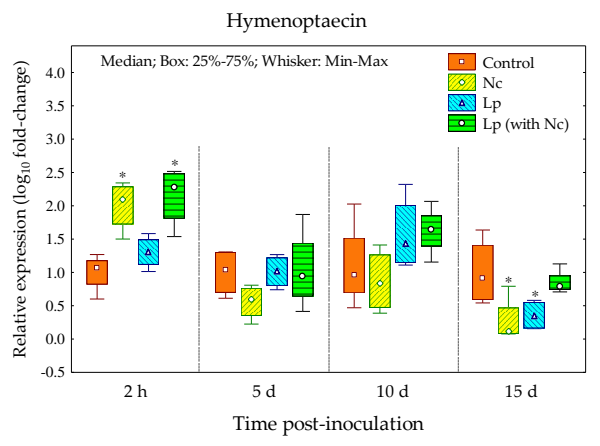

(C)

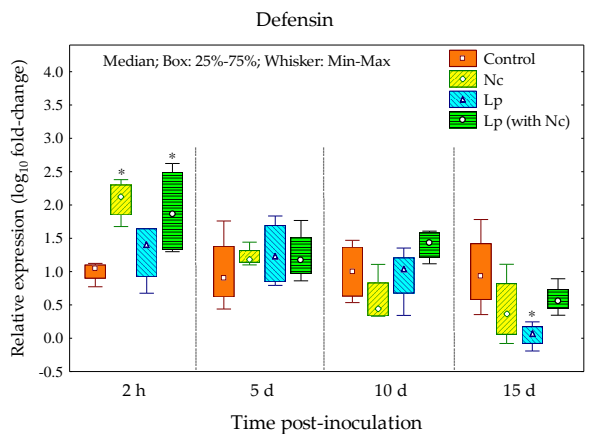

(B)

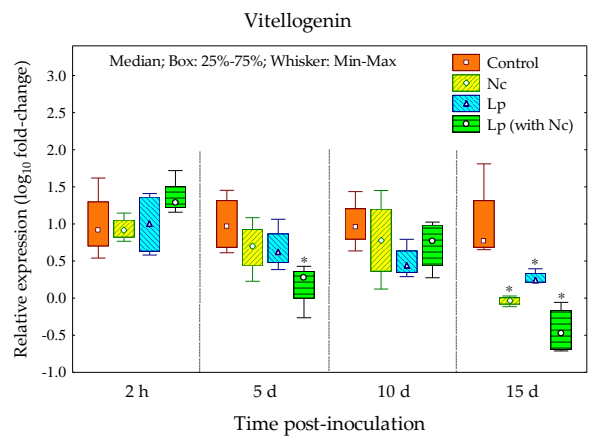

(D)

Figure 3. Box plot graphics showing the expressions (relative expressions) of genes encoding the antimicrobial peptides abaecin (A), defensin (B), hymenoptaecin (C) and vitellogenin (Vg) (D) in bees naturally infected with L. passim (Lp), healthy bees inoculated with $N$. ceranae (Nc), bees that were L. passim-infected and inoculated with $N$. ceranae ( $\mathrm{Lp}$ (with $\mathrm{Nc}$ )) and uninfected honey bees (control). Asterisks in the box plot indicate significant differences $\left(^{*}=p<0.05\right)$ with respect to the control group, according to the post hoc Dunnett test for each time post-inoculation ( $2 \mathrm{~h}$, as well as 5,10 and 15 days). 


\section{Discussion}

To the best of our knowledge, this is the first study to address the effects of infections of L. passim, $N$. ceranae and mixed infections of both pathogens considering a basal level of L. passim that bees acquired during their development in infected hives. We thus demonstrated, for the first time under controlled conditions, that mixed infections of L. passim and N. ceranae reduced bee survivals, even more so than those bees that were infected with only N. ceranae (Figure 1). This suggests that the interactions produced by mixed infections of both of these pathogens have detrimental effects on bee survivals. On the other hand, survival was not significantly affected in L. passim-infected bees. However, the distribution of the survival curve of L. passim-infected bees began to drop below that of the control group only after 15-18 dpi (Figure 1), a time period in which the L. passim load was higher than the previous measures (Figure 2A). These results indicate that L. passim does not induce a rapid death of the host compared with $N$. ceranae, although this assertion should be taken with caution, considering that the initial levels of L. passim in infected bees were lower $\left(1.0 \pm 0.6 \times 10^{3}\right.$ cell per bee) than the levels of $N$. ceranae $\left(1.0 \pm 0.3 \times 10^{5}\right.$ spores per bee) that healthy bees were inoculated with. However, our results were similar to a report by Liu et al. [41], who found that the accumulation of L. passim in the hindgut slightly decreased the honey bees' survival but never resulted in rapid death.

We have observed that the prevalence and loads of these pathogens vary throughout time, especially those of L. passim, which remains at low-media levels in spring-summer $\left(1.0 \times 10^{3}\right.$ to $1.0 \times 10^{5}$ cell equivalents per bee) and increases to high levels in fall-winter $\left(3.0 \times 10^{4}\right.$ to $3.0 \times 10^{11}$ cell equivalents per bee) (unpublished data). We also tested the effects of sporadic infections of N. ceranae in honey bees infected with a basal level of L. passim, considering that this could be a phenomenon that occurs in the field. Based on this hypothesis, we observed a competitive suppression of the L. passim load by N. ceranae, even when L. passim was already established prior to the arrival (inoculation) of N. ceranae (Figure 2A). This response could be explained by the fact that the initial infection levels of these pathogens were different, as was stated above. Nonetheless, this competitive effect proved to be significant only after $15 \mathrm{dpi}$ (Figure 2A); before this period, no suppression effect by N. ceranae was detected. Competitive suppression by N. ceranae is generally expected when a mixed infection occurs in the same space; thus, the pathogens may compete directly for host resources or space in the midgut, as has been reported with N. apis [42]. However, the suppression effect of N. ceranae on L. passim is interesting, considering that these two parasites inhabit different niches within the host. While N. ceranae invades and develops in the midgut [43] where its spores are released, these are then accumulated in the hindgut lumen [38]. On the other hand, L. passim is normally found in the hindgut, wherein the pathogen cells adhere to the hindgut wall, particularly among the rectal papillae (anterior rectum) and into the lower ileum [1]. Therefore, N. ceranae spore accumulations in the hindgut may alter the development of L. passim in this gut section, driving a possible competition at least for space. These results could explain, in part, the low prevalence (circa $2 \%$ ) of mixed infections of L. passim and $N$. ceranae, considering that, separately, they have shown a high prevalence in field samples carried out in, e.g., Chile and Switzerland [3,23].

We also demonstrated that honey bee innate immune systems, reflected by the genes encoding AMPs (e.g., defensin and hymenoptaecin), were activated immediately after becoming infected with $N$. ceranae; this was not the case in bees that were naturally infected with L. passim. However, this initial increased immune response appeared to be suppressed later not only in bees that were infected by one single species, such as N. ceranae, but, also, with L. passim (Figure 3). The roles of the AMPs on worker bees infected with $N$. ceranae have already been evaluated in previous studies, generating mixed results $[7,16,17,44]$. Some studies have shown the activation of gene signals encoding antimicrobial peptides, such as abaecin, defensin and hymenoptaecin, which act against $N$. ceranae infections, especially in the early stages of infection, between a few hours and a few days post-inoculation, inducing an overexpression of these associated genes [7,44]. Nonetheless, immune responses could also be suppressed once $N$. ceranae reaches a significant level of infection within the host [16,17]. We found that, at least in the cases of defensin and hymenoptaecin, gene expressions were activated 
in the first two hours post the oral inoculation of N. ceranae in healthy bees and those with L. passim. Nevertheless, we believe that the activation of defensin and hymenoptaecin gene expressions in mixed infections was not due to the combined effects of L. passim and N. ceranae but, rather, only due to the effect of $N$. ceranae alone, considering that single infections of L. passim did not cause a change in these expressions (Figure 3B,C). This quick response of defensin and hymenoptaecin (2 hpi) against the $N$. ceranae infection is an interesting result, considering that no structural development of $N$. ceranae has been observed to occur until $16 \mathrm{~h}$ after an inoculation [45]. Schwarz and Evans [7] have found similar results, also detecting changes in the expressions of defensin and hymenoptaecin in the early stages of an infection (e.g., 6 hpi) after an oral inoculation with N. ceranae spores. Therefore, it is possible that $N$. ceranae spore components could be delivered to the gut medium immediately after an inoculation, which could then elicit the honey bees' responses. These components could be effectors (effector proteins), which are secreted by pathogens in order to enter the host, establish a parasitic relationship, survive within the host's cells and protect the pathogen from the host's defense mechanisms [46,47]. Some N. ceranae effector candidates have been identified, and it has been hypothesized that these effectors play an important role in the $N$. ceranae infection process and its ability to survive within host cells [48]. There is evidence that three days after the initial host cell invasion, N. ceranae starts to replicate ventricular cells, releasing newly infectious spores $[45,49]$ that reach a significant infection level at 7 dpi [49]; at this point, the immune responses of the host could be suppressed [16,17]. We observed this phenomenon in the expression of AMPs but only at $15 \mathrm{dpi}$; no significant changes were found at 5 or 10 dpi (Figure 3). Similar responses were observed in bees that were naturally infected with L. passim, wherein the responses of three AMPs were downregulated at $15 \mathrm{dpi}$. At this point in time, L. passim reached a considerable load level $\left(1.0 \times 10^{6}\right.$ cells equivalents per bee; Figure $\left.2 \mathrm{~A}\right)$, which suggests that a low load of this trypanosome does not induce changes in AMPs profiles, or it is not detected by honey bee immunity AMPs-dependents, e.g., 5 or 10 dpi. However, in bees infected with C. mellificae (strain ATCC 30254), Schwarz and Evans [7] reported that some specific immune-related gene (e.g., Dscam and nim (1) responses may be downregulated over time, and tissue locations during the course of infection by this type of downregulation were not observed in the expression of the AMPs. This suggests that changes in AMP profiles could be associated with the trypanosome species' virulence, just as it has been demonstrated with Nosema species [16,17]. Additionally, we also expected a downregulation of AMPs in bees with mixed-species infections, considering that multiple pathogen infections may negatively affect the immune systems of the honey bees [7]; however, this response was not significant compared to the control. Therefore, mixed infections of L. passim and N. ceranae did not necessarily downregulate the expressions of AMPs; this could be due to the competition between the pathogens, which could reduce the cellular infection capabilities and, thus, stimulate the immune response of the host. However, new studies are required to test this hypothesis, including, e.g., an equal initial inoculum of both pathogens at the same and differently sequenced times in order to more precisely measure AMPs and other immune-associated gene responses.

One of the most interesting results was the decreased expression of the $\mathrm{Vg}$ gene in all three types of infected bees (including those with only L. passim, only N. ceranae and mixed-species infections), especially at $15 \mathrm{dpi}$ (Figure 3D). In this study, the suppressed Vg expression in this period was consistent with the breakdown point of the lifespan decline of infected honey bees, especially in bees infected with N. ceranae and mixed-species infections (Figure 1). We also observed that the Vg expression was significantly suppressed by mixed-species infections of L. passim and N. ceranae $5 \mathrm{dpi}$. This Vg suppression seemed to continue at $10 \mathrm{dpi}$, although not significantly with respect to the control group. Vitellogenin is a phospholipoglycoprotein that has multiple roles in honey bee health [29]. For instance, vitellogenin can reduce oxidative stress by scavenging for free radicals, thereby prolonging the lifespans of worker and queen bees [28]. This antioxidant activity of vitellogenin is essential to reduce the immune senescence effect on honey bees [26]. Therefore, a suppression of Vg expression could induce rapid cellular senescence, leading to a shortened lifespan in worker bees, an outcome that was observed in this study, especially in bees infected with N. ceranae and mixed-species infections. 
The differential responses found in this study of genes encoding AMPs and vitellogenin against $N$. ceranae infections, especially over the time of the study and compared with other previously reported results under controlled conditions $[7,16,17]$, could be associated with $N$. ceranae genetic variability [50], the host genotype [51] or spore doses [31]. Even the availability and quality (pollen quality) of nutritional sources could cause interference with bees' responses to $N$. ceranae infections [52] when bees used in bioassays come from well-nourished colonies. In contrast, the impacts of L. passim on AMPs and $\mathrm{Vg}$ expressions in infected bees were almost unknown until now. For the first time, we showed that L. passim may affect honey bee health, including effects on the immunity and bee survival, at least when it is mixed with other gut parasites. Notwithstanding this, the effects of L. passim infections on honey bee health at an individual and colony level, and in association with viral diseases and varroa mite parasites, are still unclear. Therefore, new studies are required to understand the dynamics of this trypanosome over the course of time and under different field conditions, with the aim of determining whether L. passim could be a newly contributing factor to colony losses in Chile and around the world.

\section{Conclusions}

In summary, we found a competitive suppression between gut parasites since the L. passim load was significantly affected by the presence of N. ceranae. However, honey bees infected with N. ceranae or with mixed infections of L. passim and N. ceranae had significantly lower survival rates than the control group. Also, the expression of the AMPs (abaecin, defensin and hymenoptaecin) and vitellogenin decreased drastically in bees with both single and mixed infections and when these pathogens reach high levels. The decrease in the expression of AMPs and vitellogenin throughout this period was consistent with the reduced survivals observed in this study, indicating that mixed infections of L. passim and N. ceranae, and even into a scenario of competition between them, may have a synergic effect on the survival and immune-related gene expressions of worker bees.

Supplementary Materials: The following are available online at http://www.mdpi.com/2075-4450/11/7/420/s1, Table S1: The health status of colonies before the experimental study in laboratory conditions.

Author Contributions: N.A. conceived this research and designed experiments; S.C., M.P.C., G.R. and T.V. performed the experiments and analysis and N.A. and M.V. wrote the paper and participated in the revisions of it. All authors have read and agreed to the published version of the manuscript.

Funding: This study was supported by Grant FONDECYT N ${ }^{\circ} 11170088$ from the National Research and Development Agency (ANID, Ex-CONICYT), Chile.

Conflicts of Interest: The authors declare that they have no conflicts of interest.

\section{References}

1. Schwarz, R.S.; Bauchan, G.R.; Murphy, C.A.; Ravoet, J.; de Graaf, D.C.; Evans, J.D. Characterization of two species of Trypanosomatidae from the honey bee Apis mellifera: Crithidia mellificae Langridge and McGhee, 1967 and Lotmaria passim n. gen., n. sp. J. Eukaryot. Microbiol. 2015, 62, 567-583. [CrossRef]

2. Arismendi, N.; Bruna, A.; Zapata, N.; Vargas, M. PCR-specific detection of recently described Lotmaria passim (Trypanosomatidae) in Chilean apiaries. J. Invertebr. Pathol. 2016, 134, 1-5. [CrossRef] [PubMed]

3. Vargas, M.; Arismendi, N.; Riveros, G.; Zapata, N.; Bruna, A.; Vidal, M.; Rodrigues, M.; Gerding, M. Viral and intestinal diseases detected in Apis mellifera in Central and Southern Chile. Chilean J. Agricult. Res. 2017, 77, 243-249. [CrossRef]

4. Castelli, L.; Branchiccela, B.; Invernizzi, C.; Tomasco, I.; Basualdo, M.; Rodriguez, M.; Zunino, P.; Antúnez, K. Detection of Lotmaria passim in Africanized and European honey bees from Uruguay, Argentina and Chile. J. Invertebr. Pathol. 2019, 160, 95-97. [CrossRef] [PubMed]

5. Runckel, C.; Flennikenm, M.L.; Engel, J.C.; Ruby, J.G.; Ganem, D.; Andino, R.; DeRisi, J.L. Temporal analysis of the honeybee microbiome reveals four novel viruses and seasonal prevalence of known viruses, Nosema, and Crithidia. PLoS ONE 2011, 6, e20656. [CrossRef] [PubMed] 
6. Ravoet, J.; Maharramov, J.; Meeus, I.; De Smet, L.; Wenseleers, T.; Smagghe, G.; de Graaf, D.C. Comprehensive bee pathogen screening in Belgium reveals Crithidia mellificae as a new contributory factor to winter mortality. PLoS ONE 2013, 8, e72443. [CrossRef] [PubMed]

7. Schwarz, R.S.; Evans, J.D. Single and mixed-species trypanosome and microsporidia infections elicit distinct, ephemeral cellular and humoral immune responses in honeybees. Dev. Comp. Immunol. 2013, 40, 300-310. [CrossRef] [PubMed]

8. Strobl, V.; Yañez, O.; Straub, L.; Albrecht, M.; Neumann, P. Trypanosomatid parasites infecting managed honeybees and wild solitary bees. Int. J. Parasitol. 2019, 49, 605-613. [CrossRef]

9. Higes, M.; Martín-Hernández, R.; Garrido-Bailón, E.; González-Porto, A.V.; García-Palencia, P.; Meana, A.; Del Nozal, M.J.; Mayo, R.; Bernal, J.L. Honeybee colony collapse due to Nosema ceranae in professional apiaries. Environ. Microbiol. Rep. 2009, 1, 110-113. [CrossRef]

10. Botías, C.; Martín-Hernández, R.; Barrios, L.; Meana, A.; Higes, M. Nosema spp. infection and its negative effects on honeybees (Apis mellifera iberiensis) at the colony level. Vet. Res. 2013, 44, 25. [CrossRef]

11. Toplak, I.; Jamnikar Ciglenečki, U.; Aronstein, K.; Gregorc, A. Chronic bee paralysis virus and Nosema ceranae experimental co-infection of winter honeybee workers (Apis mellifera L.). Viruses 2013, 5, 2282-2297. [CrossRef] [PubMed]

12. Zheng, H.Q.; Gong, H.R.; Huang, S.K.; Sohr, A.; Hu, F.L.; Chen, Y.P. Evidence of the synergistic interaction of honeybee pathogens Nosema ceranae and deformed wing virus. Vet. Microbiol. 2015, 177, 1-6. [CrossRef] [PubMed]

13. Higes, M.; Martín-Hernández, R.; Meana, A. Nosema ceranae, a new microsporidian parasite in honeybees in Europe. J. Invertebr. Pathol. 2006, 92, 93-95. [CrossRef]

14. Huang, W.F.; Jiang, J.H.; Chen, Y.W.; Wang, C.H. A Nosema ceranae isolate from the honeybee Apis mellifera. Apidologie. 2007, 38, 30-37. [CrossRef]

15. Araneda, X.; Cumian, M.; Morales, D. Distribution, epidemiological characteristics and control methods of the pathogen Nosema ceranae Fries in honeybees Apis mellifera L. (Hymenoptera, Apidae). Arch. Med. Vet. 2015, 47, 129-138. [CrossRef]

16. Antúnez, K.; Martín-Hernández, R.; Prieto, L.; Meana, A.; Zunino, P.; Higes, M. Immune suppression in the honeybee (Apis mellifera) following infection by Nosema ceranae (Microsporidia). Environ. Microbiol. 2009, 11, 2284-2290. [CrossRef]

17. Chaimanee, V.; Chantawannakul, P.; Chen, Y.; Evans, J.D.; Pettis, J.S. Differential expression of immune genes of adult honeybee (Apis mellifera) after inoculated by Nosema ceranae. J. Insect Physiol. 2012, 58, 1090-1095. [CrossRef]

18. Goblirsch, M.; Huang, Z.Y.; Spivak, M. Physiological and behavioral changes in honeybees (Apis mellifera) induced by Nosema ceranae infection. PLoS ONE 2013, 8, e58165. [CrossRef]

19. Rigaud, T.; Perrot-Minnot, M.J.; Brown, M.J. Parasite and host assemblages: Embracing the reality will improve our knowledge of parasite transmission and virulence. Proc. R. Soc. Lond. 2010, 277, 3693-3702. [CrossRef]

20. Schmid-Hempel, P. Parasites and social insects. Apidologie 1995, 26, 255-271. [CrossRef]

21. Cox, F.E.G. Concomitant infections, parasites and immune responses. Parasitology 2001, 122, S23-S38. [CrossRef] [PubMed]

22. Charbonneau, L.R.; Hillier, N.K.; Rogers, R.E.; Williams, G.R.; Shutler, D. Effects of Nosema apis, N. ceranae, and coinfections on honeybee (Apis mellifera) learning and memory. Sci. Rep. 2016, 6, 22626. [CrossRef]

23. Tritschler, M.; Retschnig, G.; Yañez, O.; Williams, G.R.; Neumann, P. Host sharing by the honeybee parasites Lotmaria passim and Nosema ceranae. Ecol. Evol. 2017, 7, 1850-1857. [CrossRef] [PubMed]

24. Evans, J.D. Beepath: An ordered quantitative-PCR array for exploring honeybee immunity and disease. J. Invertebr. Pathol. 2006, 93, 135-139. [CrossRef] [PubMed]

25. Danihlík, J.; Aronstein, K.; Petřivalský, M. Antimicrobial peptides: A key component of honeybee innate immunity. J. Apic. Res. 2015, 54, 123-136. [CrossRef]

26. Amdam, G.V.; Aase, A.L.T.; Seehuus, S.C.; Fondrk, M.K.; Norberg, K.; Hartfelder, K. Social reversal of immunosenescence in honeybee workers. Exp. Gerontol. 2005, 40, 939-947. [CrossRef]

27. Amdam, G.V.; Simões, Z.L.P.; Hagen, A.; Norberg, K.; Schrøder, K.; Mikkelsen, O.; Kirkwood, T.B.; Omholt, S.W. Hormonal control of the yolk precursor vitellogenin regulates immune function and longevity in honeybees. Exp. Gerontol. 2004, 39, 767-773. [CrossRef] 
28. Seehuus, S.C.; Norberg, K.; Gimsa, U.; Krekling, T.; Amdam, G.V. Reproductive protein protects sterile honeybee workers from oxidative stress. Proc. Natl. Acad. Sci. USA 2006, 103, 962-967. [CrossRef]

29. Amdam, G.V.; Ihle, K.E.; Page, R.E. Regulation of honeybee (Apis mellifera) life histories by vitellogenin. In Hormones, Brain and Behavior; Pfaff, D., Arnold, A., Etgen, A., Fahrbach, S., Rubin, R., Eds.; Academic Press: San Diego, CA, USA, 2009; pp. 1003-1027.

30. Dainat, B.; Evans, J.D.; Chen, Y.P.; Gauthier, L.; Neumann, P. Dead or alive: Deformed wing virus and Varroa destructor reduce the life span of winter honeybees. Appl. Environ. Microbiol. 2012, 78, 981-987. [CrossRef]

31. Antúnez, K.; Mendoza, Y.; Santos, E.; Invernizzi, C. Differential expression of vitellogenin in honeybees (Apis mellifera) with different degrees of Nosema ceranae infection. J. Apicult. Res. 2013, 52, 227-234. [CrossRef]

32. Schwarz, R.S.; Moran, N.A.; Evans, J.D. Early gut colonizers shape parasite susceptibility and microbiota composition in honeybee workers. Proc. Natl. Acad. Sci. USA 2016, 113, 9345-9350. [CrossRef] [PubMed]

33. Arismendi, N.; Castro, M.P.; Vargas, M.; Zapata, C.; Riveros, G. The trypanosome Lotmaria passim prevails in honeybees of different ages and stages of development. J. Apicult. Res. 2020.

34. Fries, I.; Chauzat, M.P.; Chen, Y.P.; Doublet, V.; Genersch, E.; Gisder, S.; Higes, M.; McMahon, D.P.; Martin-Hernandez, R.; Natsopoulou, M.; et al. Standard methods for Nosema research. J. Apicult. Res. 2013, 52. [CrossRef]

35. Porrini, M.P.; Garrido, P.M.; Eguaras, M.J. Individual feeding of honeybees: Modification of the Rinderer technique. J. Apicult. Res. 2013, 52, 194-195. [CrossRef]

36. Arismendi, N.; Vargas, M.; Lopéz, M.D.; Barría, Y.; Zapata, N. Promising antimicrobial activity against the honeybee parasite Nosema ceranae by methanolic extracts from Chilean native plants and propolis. J. Apicult. Res. 2018, 57, 522-535. [CrossRef]

37. Pfaffl, M.W. Relative quantification. In Real-time PCR, BIOS Advanced Methods; Dorak, T., Ed.; Taylor and Francis: New York, NY, USA, 2006; pp. 63-82.

38. Huang, W.; Solter, L.F. Comparative development and tissue tropism of Nosema apis and Nosema ceranae. J. Invertebr. Pathol. 2013, 113, 35-51. [CrossRef]

39. Yang, X.; Cox-Foster, D.L. Impact of an ectoparasite on the immunity and pathology of an invertebrate: Evidence for host immunosuppression and viral amplification. Proc. Natl. Acad. Sci. USA 2005, 21,7470-7475. [CrossRef]

40. Holm, S. A simple sequential rejective method procedure. Scand. J. Stat. 1979, 6, 65-70.

41. Liu, Q.; Lei, J.; Darby, A.C.; Kadowaki, T. Trypanosomatid parasite dynamically changes the transcriptome during infection and modifies honeybee physiology. Commun. Biol. 2020, 3, 1-8. [CrossRef]

42. Natsopoulou, M.E.; McMahon, D.P.; Doublet, V.; Bryden, J.; Paxton, R.J. Interspecific competition in honeybee intracellular gut parasites is asymmetric and favors the spread of an emerging infectious disease. Proc. $R$. Soc. B 2015, 282, 282. [CrossRef]

43. Chen, Y.P.; Evans, J.D.; Murphy, C.; Gutell, R.; Zuker, M.; Gundensen-Rindal, D.A.; Pettis, J.S. Morphological, molecular, and phylogenetic characterization of Nosema ceranae, a microsporidian parasite isolated from the European honey bee, Apis mellifera. J. Eukaryot. Microbiol. 2009, 56, 142-147. [CrossRef] [PubMed]

44. Huang, Q.; Chen, Y.P.; Wang, R.W.; Cheng, S.; Evans, J.D. Host-parasite interactions and purifying selection in a Microsporidian parasite of honeybees. PLOS ONE 2016, 11, e0147549.

45. Gisder, S.; Möckel, N.; Linde, A.; Genersch, E. A cell culture model for Nosema ceranae and Nosema apis allows new insights into the life cycle of these important honeybee-pathogenic microsporidia. Environ. Microbiol. 2011, 13, 404-413. [CrossRef] [PubMed]

46. Cambronne, E.D.; Roy, C.R. Recognition and delivery of effector proteins into eukaryotic cells by bacterial secretion systems. Traffic 2006, 7, 929-939. [CrossRef] [PubMed]

47. Stergiopoulos, I.; de Wit, P.J.G.M. Fungal effector proteins. Annu. Rev. Phytopathol. 2009, 47, $233-263$. [CrossRef] [PubMed]

48. Lalik, M. Characterization of secreted effector proteins of Nosema ceranae, an agent associated with colony collapse disorder (CCD). Ph.D. Thesis, University of Exeter, Exeter, UK, January 2015.

49. Higes, M.; García-Palencia, P.; Martín-Hernández, R.; Meana, A. Experimental infection of Apis mellifera honeybees with the Microsporidia Nosema ceranae. J. Invertebr. Pathol. 2007, 94, 211-217. [CrossRef]

50. Medici, S.K.; Sarlo, E.G.; Porrini, M.P.; Braunstein, M.; Eguaras, M.J. Genetic variation and widespread dispersal of Nosema ceranae in Apis mellifera apiaries from Argentina. Parasitol. Res. 2012, 110, 859-864. [CrossRef] 
51. Gregorc, A.; Silva-Zacarin, E.C.; Carvalho, S.M.; Kramberger, D.; Teixeira, E.W.; Malaspina, O. Effects of Nosema ceranae and thiametoxam in Apis mellifera: A comparative study in Africanized and Carniolan honeybees. Chemosphere 2016, 147, 328-336. [CrossRef]

52. Di Pasquale, G.; Salignon, M.; Le Conte, Y.; Belzunces, L.P.; Decourtye, A.; Kretzschmar, A.; Suchail, S.; Brunet, J.L.; Alaux, C. Influence of pollen nutrition on honeybee health: Do pollen quality and diversity matter? PLoS ONE 2013, 8, e72016. [CrossRef]

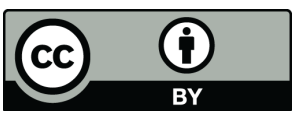

(C) 2020 by the authors. Licensee MDPI, Basel, Switzerland. This article is an open access article distributed under the terms and conditions of the Creative Commons Attribution (CC BY) license (http://creativecommons.org/licenses/by/4.0/). 